\title{
Brief des Schriftleiters an die Leser
}

\section{J.-Matthias Graf von der Schulenburg}

Online publiziert: 12. Dezember 2018

(C) Springer-Verlag GmbH Deutschland, ein Teil von Springer Nature 2018

Vor 20 Jahren übergab mir Dieter Farny die Schriftleitung der Zeitschrift für die gesamte Versicherungswissenschaft (ZVersWiss). Ende diesen Jahres gebe ich das Amt weiter. Die Schriftleitung wird dann von der Geschäftsstelle des deutschen Vereins für Versicherungswissenschaften und einem interdisziplinären Schriftleitergremium übernommen.

Die ZVersWiss ist mit 107 Jahren meines Wissens die älteste versicherungswissenschaftliche Zeitschrift der Welt. Der „Marktführer“, The Journal of Risk and Insurance (JRI), ist z. B. „nur“ 85 Jahre alt. Seit der Gründung der ZVersWiss bin ich der fünfte Schriftleiter, was die große Kontinuität der Zeitschrift und die „Langlebigkeit" der Schriftleiter der ZVersWiss verdeutlicht.

Auf die letzten 20 Jahre schaue ich mit tiefer Dankbarkeit zurück. Es war ein großes Privileg, die Arbeit meiner Vorgänger fortzuführen. Besonders danke ich meiner Schriftleitungsassistentin, Frau Dr. Ute Lohse, für ihren großen Einsatz und ihre sorgfältige und umsichtige Arbeit. Dem Deutschen Verein für Versicherungswissenschaften e. V. (DVfVW) - und hier stellvertretend der Geschäftsführerin des Vereins, Frau Dr. Andrea Uber - danke ich für das Vertrauen und die stete großzügige Unterstützung der Zeitschrift. Auch gilt mein herzlicher Dank den Autoren, den Gutachtern, den Bereichsschriftleitern und dem Beirat, die mit Rat und Tat meine Arbeit unterstützt haben.

Die ZVersWiss ist nicht nur die älteste, sondern auch die führende deutschsprachige versicherungswissenschaftliche Zeitschrift. Sie hatte immer bewusst das Profil einer interdisziplinären Zeitschrift gepflegt, da der DVfVW die Vereinigung aller Versicherungswissenschaftler ist und die Beantwortung vieler versicherungswissenschaftlicher Fragen, Kenntnisse in mehreren Disziplinen erfordert. Wer sich

J.-M. Graf von der Schulenburg ( $ه)$

Institut für Versicherungsbetriebslehre, Leibniz Universität Hannover, Hannover, Deutschland

E-Mail:.jms@ivbl.uni-hannover.de 
beispielsweise über den Versicherungsvertrieb Gedanken macht, muss sowohl ökonomische Kenntnisse haben als auch die Vorgaben des Versicherungsvertragsrechts beachten. Wer sich mit der Produktgestaltung und -optimierung in der Lebens- und Krankenversicherung beschäftigt - um ein anderes Beispiel zu nennen - muss neben betriebswirtschaftlichen Kenntnissen auch Wissen in Versicherungsmathematik und Versicherungsmedizin haben.

Der Nachteil einer interdisziplinären Zeitschrift ist jedoch, dass sie in disziplinären Rankings, die heutzutage z. B. in der ökonomischen Forschung zunehmende Bedeutung haben, nicht so erfolgreich sein kann wie eine rein disziplinäre Zeitschrift: Beim Scientific Journal Ranking (SJR) hat die ZVersWiss Werte von 0,2 bis 0,3 und im VHB-Ranking ein C bzw. D. Das JRI hat hingegen ein SJR von 1,4 und einen Impact Factor von 1,343, und ist damit im ISI-Ranking auf Platz 38 der 96 Business und Finance Zeitschriften und auf Platz 110 der 347 EconomicsZeitschiften (2016). Dennoch habe ich mich aus verschiedenen Gründen immer dafür eingesetzt, dass die Zeitschrift vorwiegend deutschsprachig bleibt und allen Disziplinen der Versicherungswissenschaft als Forum dient. In der Zeitschrift publizieren nicht nur Fachwissenschaftler, die sich international profilieren wollen, sondern auch Wissenschaftler aller Hochschulen und wissenschaftlich arbeitende Praktiker. Die Leserschaft umfasst Wissenschaftler sowie Praktiker, die breit über die wissenschaftlichen Themen informiert werden wollen. Zudem gibt es Fragestellungen, die sich besonders auf Deutschland beziehen. Eine Stärke der ZVersWiss war auch, dass sie sich auch mit Herausforderungen der Sozialversicherung sowie den Interdependenzen zwischen Sozial- und Individualversicherung auseinandersetzt.

Trotz der oben beschriebenen Kontinuität gab es in den letzten 20 Jahren einige Veränderungen, auf die ich hinweisen möchte: Gemäß den heute üblichen Standards bei wissenschaftlichen Zeitschriften wurde ein doppelt-blinder Gutachterprozess eingeführt. Um den versicherungswissenschaftlichen Disziplinen gerecht zu werden, wurden vier Bereichsschriftleiter (Co-Editors) für die Disziplinen Versicherungsrecht, -mathematik und -medizin sowie Sozialversicherung berufen: Manfred Wandt, Angelika May, Stephan Becher und Heinz-Dietrich Steinmeyer. Daneben wurde ein interdisziplinär und international besetzter Beirat (Associate Editors) etabliert. Derzeit gehören dem Beirat Peter Albrecht, Christian Armbrüster, Meinrad Dreher, Randy E. Dumm, Louis Eeckhoudt, Christian Hipp, Norbert Klusen, Klaus E. Schmidt und Peter Zweifel an. Die Beiratsmitglieder Harris Schlesinger und Paul Kleindorfer sind leider in den letzten Jahren verstorben.

Weitere bedeutende Änderungen betrafen den Verlagswechsel zu Springer, die Modernisierung des Formats und des Layouts der Zeitschrift wie auch die Verfügbarkeit übers Internet und die „online first“-Veröffentlichung aller angenommenen Aufsätze. Außerdem wurden alle früheren Aufsätze digitalisiert, sodass sie der Wissenschaft leichter zugänglich sind.

Die ZVersWiss erscheint derzeit in 5 Heften pro Jahr mit insgesamt ca. 630 Seiten. Eins der Hefte ist immer ein Sonderheft mit Beiträgen der letzten Jahrestagung des Deutschen Vereins für Versicherungswissenschaften e. V. Es enthält sowohl die Plenumsvorträge als auch ausgewählte Forums-Beiträge junger Wissenschaftler.

Die zukünftige Schriftleitung wird die ZVersWiss weiter entwickeln und u. a. vor folgenden Fragen stehen: 
- Welche Sprache soll die ZVersWiss haben: deutsch - mit der Möglichkeit auch englisch zu publizieren - (status quo) oder durchgehend englisch?

- Soll die ZVersWiss zukünftig Schwerpunkthefte haben?

- Soll die ZVersWiss interdisziplinär bleiben (status quo) oder auch einen disziplinären Teil erhalten - wie z. B. ein ,open access journal“ für Ökonomen?

- Soll die ZVersWiss ein Printmedium mit online first Publikation bleiben oder in ein „open access journal“ transformiert werden?

- Wie soll das Schriftleitungsgremium strukturiert und besetzt sein?

Bei der Lösung dieser und anderer Fragen wünsche ich meinen Nachfolgern viel Erfolg. Vor allem wünsche ich Ihnen so viel Spaß bei der wunderbaren Schriftleitungs-Arbeit, wie ich ihn in den letzten 20 Jahren gehabt habe.

J.-Matthias Graf von der Schulenburg

Hannover, im November 2018 